RESEARCH REPORT

\title{
Association of elevated preoperative blood pressure with preincision hypotension in pediatric surgical patients
}

\author{
Margaret Stewart, Joseph Scattoloni, Golshid Tazhibi \& Olubukola O. Nafiu \\ Department of Anesthesiology, Section of Pediatric Anesthesiology, University of Michigan, Ann Arbor, MI, USA
}

\section{What is already known}

- High blood pressure is increasingly prevalent in the pediatric population highlighting the need for children to be screened for this condition during health care visits. Hypertension is an established risk factor for long-term overall poor health outcome in adults and children.

\section{What this article adds}

- We provide data for the first time in children which showed a high prevalence of preoperative elevated blood pressure and its strong association with preincision hypotension.

\section{Keywords}

children and adolescents; prehypertension; hypertension; preincision hypotension; overweight; obesity

\section{Correspondence}

Olubukola O. Nafiu, University of Michigan Health System, 1500 East Medical Centre Drive, Room UH 1H247, Ann Arbor, MI 48109-0048, USA

Email: onafiu@med.umich.edu

Section Editor: Laszlo Vutskits

Accepted 10 May 2016

doi:10.1111/pan.12945

\section{Summary}

Introduction: High blood pressure (BP) in childhood is associated with left ventricular hypertrophy and abnormal vascular reactivity even in apparently healthy children. This study examined the prevalence of high BP in children undergoing elective noncardiac operations. We also determined the association of preoperative high BP with preincision hypotension (PIH).

Methods: This was a retrospective cohort study that used clinical and anthropometric data on children aged 3-17 years who underwent elective, noncardiac operations from January 2006 to January 2014. Preoperative blood pressure (BP) was used to stratify children into three categories: normal BP (systolic and diastolic BP below the 90th percentile), prehypertension (systolic and/or diastolic BP $\geq 90$ th percentile but $<95$ th percentile or if the BP exceeds $120 / 80 \mathrm{mmHg}$ even if it is below the 90th percentile), and hypertension (systolic and/or diastolic $\mathrm{BP} \geq 95$ th percentile). Multivariable logistic regression analysis was used to calculate adjusted odds ratios for PIH using age, gender, body mass index (BMI), and BP categories as covariates.

Results: Among 35832 children, the overall prevalence of prehypertension, hypertension, and hypertension by adult standards were $16.4 \%, 6.8 \%$, and $3.3 \%$, respectively. Overweight and obese children had higher rates of elevated BP. Presence of elevated baseline BP, high BMI category, and presence of OSA diagnosis were independent predictors of multiple episodes of $\mathrm{PIH}$ in a logistic regression model.

Conclusion: In children, preoperative high BP is an independent predictor of PIH. Although the longtime consequences of high BP are well known, this report provides the first intraoperative evidence of adverse event associated with preoperative high BP in children. Mechanisms underlying this hypotensive response are unclear. 


\section{Introduction}

High blood pressure (BP) is increasingly prevalent among children and adolescents in the United States (1-3). Current estimates are that about $2-5 \%$ of children have a diagnosis of hypertension (4). Although many of the well-described consequences of high BP such as stroke, myocardial infarction, and heart failure are uncommon in children, hypertension (HT) in childhood may lead to HT in adulthood and hypertensive children often manifest features of target organ damage, such as left ventricular hypertrophy, diastolic dysfunction (5), and atherosclerosis (6). HT is also an independent risk factor for lower cognitive function especially in obese individuals (7) and more recently primary HT in children and adolescents was associated with a higher rate of learning disabilities (8). Because chronically elevated $\mathrm{BP}$ can result in decreased systemic health over the long term, it is imperative to identify and treat children with high BP. However, it appears that physicians and other health care workers frequently overlook high BP in children (9). Consequently, the American Academy of Pediatrics (AAP) and the American Heart Association National High Blood Pressure Education Program (NHBPEP) recommends that children $\geq 3$ years old who are seen in medical care settings should have their BP measured at least once during every health care visit (10). Indeed, routine monitoring of BP during anesthesia is one of the recommended standards of care by the American Society of Anesthesiologists (ASA) (11).

In adult patients, undiagnosed or untreated elevated $\mathrm{BP}$ is considered a risk factor for perioperative complications including, labile BP trends (hypo- and/or hypertension), myocardial ischemia, and perioperative stroke (13-16). These complications are especially more frequent following induction of general anesthesia and during laryngoscopy (15).

Although the preceding complications are vanishingly rare in pediatric surgical patients, the prevalence and consequences of elevated preoperative BP have not been comprehensively studied, with most published data only relevant to adult patients (16). Previous reports of intraoperative hypotension in children have not considered the prevalence of baseline elevated BP or its possible association with postinduction hypotension (17).

Because the preoperative review represents a health care visit during which clinical and anthropometric data are routinely collected, this encounter could be leveraged for screening children for elevated BP. Therefore, this study determined the prevalence of high BP (prehypertension and hypertension) in children undergoing elective noncardiac operations. We also examined whether children with preoperative elevated $\mathrm{BP}$ were at increased risk for preincision hypotension (PIH). The hypothesis tested was that, preoperative elevated $\mathrm{BP}$ would be associated with increased frequency of PIH.

\section{Methods}

Following Institutional Review Board (IRB) approval (HUM00068846), we acquired data from our electronic perioperative medical information system (Centricity ${ }^{\circledR}$; General Electric Healthcare, Waukesha, WI, USA). This was a retrospective cohort study that utilized clinical and anthropometric data on children aged 3-17 years who underwent elective, noncardiac operations from January 2006 to January 2014. We only included ages down to 3 year because the AAP recommends routine BP screening for children $\geq 3$ year (10). The following information were extracted from the database: age, gender, ethnicity, American Society of Anesthesiology (ASA) status, surgical specialty, as well as height and weight. Body mass index (BMI) was calculated as weight in kilograms divided by the square of the height in meters $\left(B M I=\mathrm{kg} \cdot \mathrm{m}^{-2}\right.$ ). Children were then classified as normal weight (BMI $<85$ th percentile), overweight (BMI $\geq 85$ th and $<95$ th percentile), or obese (BMI $\geq 95$ th percentile) using age- and gender-specific reference growth charts (19) from the National Center for Health Statistics (NCHS)/Centers for Disease Control and Prevention (CDC).

\section{Blood pressure data and definitions of elevated BP and hypotension}

Blood pressure measurements were recorded on all patients during the normal course of preanesthesia evaluation. Standard nursing BP protocols were used. Typically, an experienced preoperative care nurse or a suitably trained medical assistant measures the patient's BP using an oscillometric machine and an appropriately sized BP cuff. Clinical and hemodynamic variables are typically measured prior to the administration of premedication thus reducing the possible effect of premedication on the BP values used for the present study.

\section{Case ascertainment}

Systolic and diastolic BP (SBP and DBP) were used to identify children with elevated BP. We defined normal BP as height-, age-, and sex-specific systolic and diastolic BP below the 90th percentile. We used the 50th percentile of height value in the abnormal BP percentile algorithm recommended by the US National High Blood Pressure Education Program Working Group 
(10). Prehypertension (PHT) indicates systolic and/or diastolic $\mathrm{BP} \geq 90$ th percentile but $<95$ th percentile or if the BP exceeds $120 / 80 \mathrm{mmHg}$ even if it is below the 90th percentile. Hypertension (HT) denotes systolic and/or diastolic BP $\geq 95$ th percentile for age, sex, and height (10). We also categorized each child's SBP and DBP as being above or below $140 \mathrm{mmHg}$ and $90 \mathrm{mmHg}$, respectively (the threshold for adult hypertension). This we classified as, 'Obviously elevated BP'.

\section{Control ascertainment}

Inclusion criteria for controls were all children aged 317 years with normal preoperative BP who underwent elective operations during the study period. No systematic matching of control to cases was performed.

\section{Preincision hypotension (PIH)}

The procedure for calculating and defining PIH was fully described in our prior publication (18) but is briefly reviewed here. Intraoperative blood pressure (BP) measurement was done automatically using either the oscillometric method. Intraoperative BP data were acquired from an automated, electronic physiologic monitor (Solar 9500; General Electric Healthcare). BP values were automatically recorded every $3 \mathrm{~min}$ for every patient. Each intraoperative anesthetic record was divided into successive 5-min epochs. The median SBP for each 5-min epoch on the anesthesia chart was then calculated. The use of a median value over a defined time period filters out monitoring artifacts and clinically insignificant, transient hypotension (19). The number of SBP epochs below defined cutoffs during the preincision period was computed for every patient and compared with age-dependent cutoff points for hypotension: age below 10 years, hypotension is SBP lower than $2 n+70 \mathrm{mmHg}$ (where $n=$ age in years); for children older than 10 years, hypotension equals SBP $<90 \mathrm{mmHg}$ (20). Children having two or more episodes of PIH were classified as having multiple episodes of hypotension. Finally, administration of ephedrine, calcium gluconate, or phenylephrine in the preincision period was taken as proxy for hypotension requiring intervention ('clinically important hypotension') - as these drugs are not usually administered in the preincision period except to treat hypotension.

\section{Exclusion criteria}

Children with a preoperative history of hypertension on treatment were excluded from the present analyses. Additionally, we excluded data on children with a history of renal or liver failure. Furthermore, we excluded all emergency operations because they may be associated with pain, which could spuriously elevate the preoperative BP.

\section{Data validation}

Two principal methods were used to validate the data used for the present analysis. First, clinically implausible values for the dependent and predictor variables (e.g., BMI $>80 \mathrm{~kg} \cdot \mathrm{m}^{-2}$ or $<8 \mathrm{~kg} \cdot \mathrm{m}^{-2}$ ) were excluded. Also, children with missing preoperative $\mathrm{BP}$, height and/or weight, as well as BMI were excluded. Other variables with missing data were analyzed 'as is', i.e., only available data were analyzed without any attempt at data imputation. Only one perioperative visit per patient per year was counted and included for analyses. For children undergoing multiple surgical procedures during a 1-year period, the first visit was chosen for analysis.

\section{Statistical analysis}

Data analyses were carried out with Statistical Package for the Social Sciences (SPSS) for wINDOws, version 22.0, (SPSS Inc., Chicago, IL, USA) and SAS version 9.3. A two-tailed $P$-value $<0.05$ was considered statistically significant for all procedures.

The primary hypothesis tested was that the rates of preincision hypotension would differ based on preoperative BP category. We first examined all continuous variables (age, height, and weight, as well as BMI, SBP, and DBP) for normal distribution with the Kolmogorov-Smirnov test. All normally distributed variables should have a $P>0.05$ by this test (21). Basic descriptive statistics, including means, standard deviations, and percentages were calculated for demographic and clinical data. Crude prevalence of high BP (pre-HT and HT) were described as simple proportions and compared across BMI categories using Pearson's chi-squared test. Similarly, the proportion of children with 'obviously' elevated BP (hypertension by adult standards) was computed. Relatedly, the prevalence of overweight and obesity was described as simple proportions and compared across sex groups. Differences in the distribution of BP categories were compared across BMI groups and presented as simple bar graphs. Next, we constructed a sex-specific Pearson correlation matrix to assess the strength of association between the following continuous variables: age, height, weight, BMI, SBP, and DBP.

\section{Multivariable regression analysis}

To fit a multivariable logistic regression model, we estimated the $\operatorname{Pr}(Y=1 \mid X)$, where $Y$ is the binary-dependent variable (multiple PIH yes/no) and $X$ is the vector 
of the covariates. To derive the response variable $Y$, we calculated the number of episodes of hypotension in the preincision period and categorized two or more episodes as multiple PIH.

Prior to fitting the logistic regression model, covariates were examined for multi-collinearity by scanning for highly correlated variables $(\rho \geq 0.7)$. After collinearity was assessed, age, weight, and height were highly correlated. Because age and BMI are considered essential variables in the diagnosis of elevated BP in children (12), they were retained in the regression model while body weight and height were dropped. A model that included height, weight, and BMI displayed statistically significant Hosmer-Lemeshow $P$-value indicating poor fit to the data (data not shown). Dichotomous covariates were coded as dummy variables $(0=$ no, $1=$ yes $)$. Variables were included in the model based on statistical significance, clinical relevance, (18) and due consideration for over parameterization.

Receiver operating characteristic (ROC) and area under the curve (AUC) were used to assess the final model's predictive ability. ROC curves are typically plotted to demonstrate the discriminatory power of a diagnostic test over the entire range of test results. A good test has an ROC curve skewed to the upper left corner (22). The AUC defines the diagnostic power of a test; a perfect score has an AUC of 1, while an AUC of 0.5 means the test performs no better than chance (22).

\section{Results}

A total of 38291 records with complete clinical and anthropometric data were extracted from the database.
After excluding 2459 records due to implausible data (1646 with BMI values outside defined range and 813 with $\mathrm{BP}$ values outside normal physiologic range), the final study cohort included 35832 children. Of these, $26.5 \%$ had elevated preoperative BP while $14.3 \%$ were overweight and $15.7 \%$ were obese. The overall prevalence of PHT and hypertension and hypertension by adult standards were $16.4 \%, 6.8 \%$, and $3.3 \%$, respectively. All the indices of adiposity and BP were significantly positively correlated in both boys and girls (Table 1). Expectedly, overweight and obese children had higher rates of elevated BP (Figure 1). Boys had significantly higher odds of preoperative high BP compared to girls $(26.7 \%$ vs $21.1 \%$; OR $=1.36,95 \% \mathrm{CI}=1.29$ 1.43; $P=0.001)$.

On average, children with elevated BP were older, had higher anthropometric parameters, and were more likely to have obesity-associated diagnoses such as habitual snoring and OSA (Table 2). Those with high BP were also more likely to belong to higher ASA class. During the preincision period, $71.1 \%$ of patients received intravenous fentanyl while $59.6 \%$ received intravenous propofol. On average, there was no statistically significant difference between normotensive and those with high BP in the fentanyl dose per $\mathrm{kg}$ body weight used during the preincision period (Table 2). We also observed no significant difference in the occurrence of PIH by IV fentanyl use (high BP group $76.9 \%$ vs normotensive group $71.0 \% ; P=0.639)$.

The overall incidence of any episode of PIH was $24.5 \%$ while multiple episodes of PIH occurred in $9.8 \%$ patients. Children with elevated baseline BP had significantly higher odds of multiple PIH compared with normotensive

Table 1 Summary statistics including sex-specific Pearson's correlations, means, and standard deviation for clinical and anthropometric variables among 35832 pediatric surgical patients

\begin{tabular}{|c|c|c|c|c|c|c|c|c|}
\hline & 1 & 2 & 3 & 4 & 5 & 6 & Mean & $\mathrm{SD}$ \\
\hline \multicolumn{9}{|l|}{ Girls ( $n=16014)$} \\
\hline 1 Age (year) & 1.00 & & & & & & 9.44 & 4.38 \\
\hline 2 Weight $(\mathrm{kg})$ & 0.79 & 1.00 & & & & & 37.83 & 21.48 \\
\hline 3 Height $(\mathrm{cm})$ & 0.89 & 0.83 & 1.00 & & & & 133.65 & 24.52 \\
\hline $4 \mathrm{BMI}\left(\mathrm{kg} \cdot \mathrm{m}^{-2}\right)$ & 0.46 & 0.79 & 0.38 & 1.00 & & & 19.64 & 5.85 \\
\hline $5 \mathrm{SBP}(\mathrm{mmHg})$ & 0.38 & 0.42 & 0.39 & 0.33 & 1.00 & & 109.10 & 14.30 \\
\hline $6 \mathrm{DBP}(\mathrm{mmHg})$ & 0.27 & 0.24 & 0.24 & 0.17 & 0.58 & 1.00 & 64.26 & 9.90 \\
\hline \multicolumn{9}{|l|}{ Boys ( $n=19818$ ) } \\
\hline 1 Age (year) & 1.00 & & & & & & 9.40 & 4.39 \\
\hline 2 Weight $(\mathrm{kg})$ & 0.82 & 1.00 & & & & & 39.59 & 23.28 \\
\hline 3 Height $(\mathrm{cm})$ & 0.92 & 0.86 & 1.00 & & & & 136.61 & 27.54 \\
\hline $4 \mathrm{BMI}\left(\mathrm{kg} \cdot \mathrm{m}^{-2}\right)$ & 0.44 & 0.75 & 0.38 & 1.00 & & & 19.49 & 5.65 \\
\hline $5 \mathrm{SBP}(\mathrm{mmHg})$ & 0.49 & 0.51 & 0.48 & 0.37 & 1.00 & & 110.90 & 15.39 \\
\hline
\end{tabular}

BMI, body mass index; SBP, systolic blood pressure; DBP, diastolic blood pressure. All correlations significant at $P<0.001$. 


\section{Prevalence of high blood pressure by BMI categories}

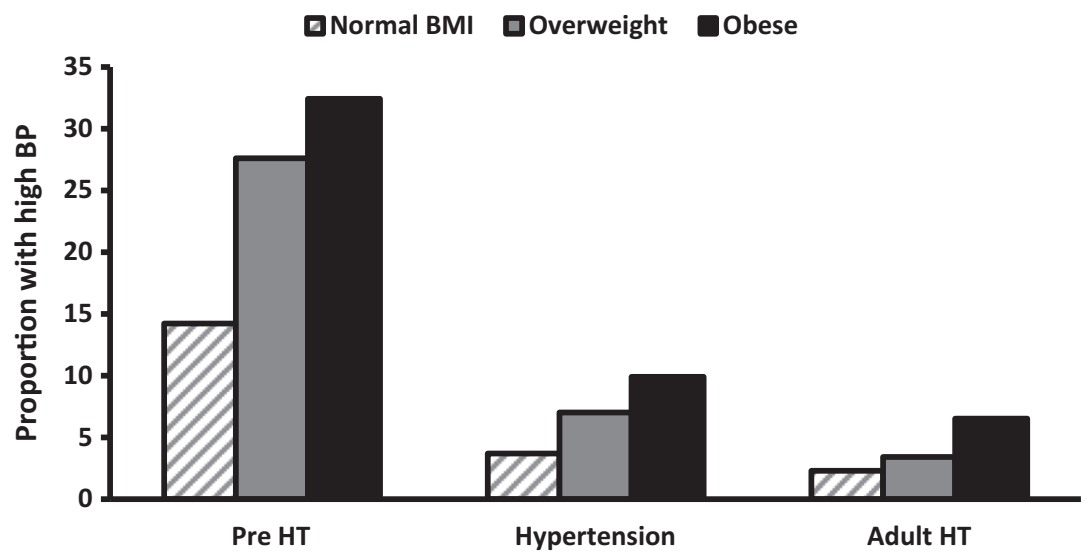

Figure 1 Prevalence of elevated BP (prehypertension, hypertension, and adult hypertension) in the study subjects stratified according to BMl categories. Prevalence of high BP was significantly $(P<0.001)$ higher in all children with increasing $\mathrm{BMI}$ groups. Abbreviations: $\quad \mathrm{BP}=$ blood $\quad$ pressure; $\mathrm{BMI}=$ body mass index .
Table 2 Baseline clinical and anthropometric features of the study cohort stratified by BP categories

\begin{tabular}{|c|c|c|c|}
\hline Variables & $\begin{array}{l}\text { Normotensive } \\
(n=27168)\end{array}$ & $\begin{array}{l}\text { High BP } \\
(n=8664)\end{array}$ & $P$-values \\
\hline \multicolumn{4}{|l|}{ Continuous, Mean \pm SD } \\
\hline Age (years) & $8.62 \pm 4.12$ & $11.92 \pm 4.06$ & $<0.001$ \\
\hline Height (cm) & $130.52 \pm 24.85$ & $150.24 \pm 24.99$ & $<0.001$ \\
\hline Weight (kg) & $34.01 \pm 18.90$ & $53.84 \pm 25.78$ & $<0.001$ \\
\hline $\mathrm{BMI}\left(\mathrm{kg} \cdot \mathrm{m}^{-2}\right)$ & $18.61 \pm 5.04$ & $22.50 \pm 6.91$ & $<0.001$ \\
\hline $\mathrm{SBP}(\mathrm{mmHg})$ & $103.79 \pm 10.23$ & $129.86 \pm 8.92$ & $<0.001$ \\
\hline $\mathrm{DBP}(\mathrm{mmHg})$ & $61.82 \pm 8.54$ & $71.77 \pm 10.34$ & $<0.001$ \\
\hline IV Fentanyl per kg & $0.02 \pm 0.01$ & $0.01 \pm 0.01$ & 0.5480 \\
\hline \multicolumn{4}{|l|}{ Categorical (\%) } \\
\hline Snoring history & 20.2 & 32.8 & $<0.001$ \\
\hline OSA history & 11.1 & 11.9 & 0.039 \\
\hline Asthma history & 34.9 & 35.8 & 0.147 \\
\hline Overweight/Obese & 20.0 & 32.0 & $<0.001$ \\
\hline ASA class III & 19.1 & 21.3 & $<0.001$ \\
\hline Inhalational induction & 65.3 & 64.1 & 0.642 \\
\hline $\begin{array}{l}\text { Muscle } \\
\text { relaxants used }\end{array}$ & 31.9 & 30.8 & 0.929 \\
\hline $\begin{array}{l}\text { Preincision } \\
\text { hypotension }\end{array}$ & 5.8 & 26.1 & $<0.001$ \\
\hline Severe hypotension & 0.5 & 3.0 & $<0.001$ \\
\hline Ephedrine $y / n$ & 0.3 & 0.5 & 0.022 \\
\hline Phenylephrine $y / n$ & 0.5 & 1.1 & $<0.001$ \\
\hline
\end{tabular}

BMI, body mass index; SBP, systolic blood pressure; DBP, diastolic blood pressure; OSA, obstructive sleep apnea; ASA, American society of anesthesiologists; IV, intravenous.

children $(26.1 \%$ vs $5.8 \%$; OR $=5.7,95 \% \mathrm{CI}=5.3-6.1$; $P<0.001)$. Increasing baseline SBP decile was significantly associated with increasing frequency of multiple PIH (Figure 2). Furthermore, children with baseline elevated SBP were more likely to develop hypotension treated with ephedrine $(0.5 \%$ vs $0.3 \% ; P=0.016)$ or phenylephrine $(1.1 \% \quad$ vs $\quad 0.5 \% ; \quad P<0.001)$ (Table 2$)$.
Finally, preincision use of muscle relaxants was not significantly different between the groups (Table 2).

Multivariate logistic regression indicated that the factors detailed in Table 3 were associated with the occurrence of multiple episodes of PIH. Model diagnostic parameters are included in the table. The ROC curve analysis of this model showed an AUC of $0.75 ; 95 \%$ $\mathrm{CI}=0.73-0.77 ; P=0.001$ (Figure 3). Elevated BP category was associated with the highest odds of PIH. Specifically, when controlling for the other covariates in the model, preoperative elevated $\mathrm{BP}$ was associated with sixfold higher odds of multiple episodes of PIH $(\mathrm{OR}=6.22 ; \quad 95 \% \quad$ confidence $\quad$ interval $=5.76-6.71$, $P<0.001)$. Furthermore, high BMI category and preoperative OSA diagnoses were associated with $22 \%$ and $49 \%$ increased odds of multiple episodes of PIH, respectively. Interestingly, age and gender were not significant predictors of multiple episodes of PIH in the final model.

\section{Discussion}

In this retrospective cross-sectional study of children and adolescents who underwent elective noncardiac operations, we found a high prevalence of elevated BP (pre-HT and HT) particularly among overweight/obese children. Indeed, the present study confirms our previous report of the association of elevated BP with obesity status in a much smaller prospective cohort of children undergoing elective surgical procedures (23) which validates the BP measurement and documentation process at our institution. We also report for the first time in a pediatric surgical population that elevated preoperative SBP was an independent risk factor for multiple episodes of PIH. Episodes of PIH were more frequent with increasing deciles of preoperative SBP. 
Figure 2 Showing increasing incidence of episodes of preincision hypotension (PIH) with increasing deciles of preoperative systolic blood pressure (SBP).

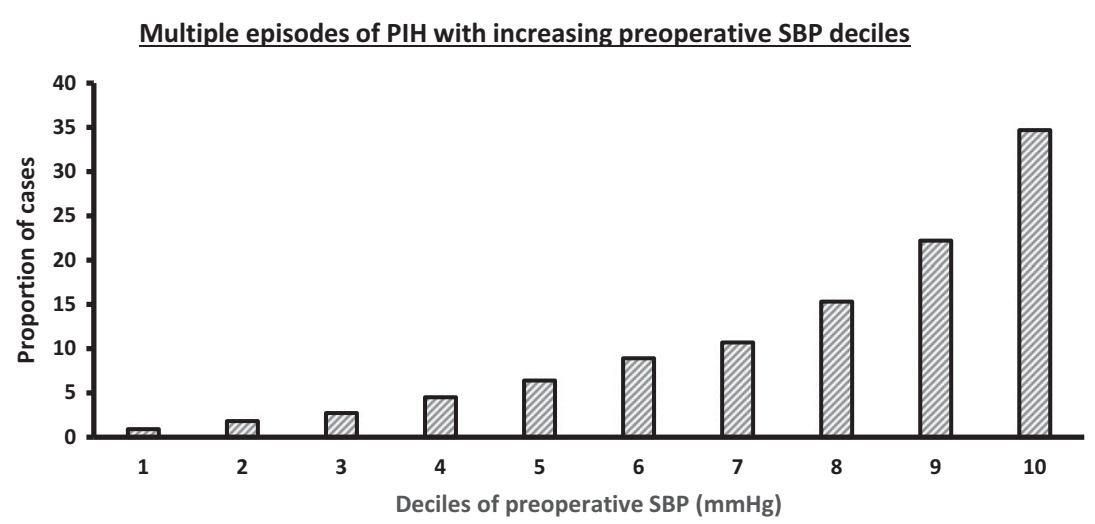

Table 3 Result of stepwise logistic regression (final model) to estimate the adjusted odds ratio for the factors associated with multiple episodes of preincision hypotension

\begin{tabular}{|c|c|c|c|}
\hline Independent predictors & OR & $95 \% \mathrm{Cl}$ & $P$-value \\
\hline \multicolumn{4}{|l|}{ All covariates included } \\
\hline Sex (girl vs boy) & 1.02 & $0.95-1.11$ & 0.536 \\
\hline Age (per year) & 1.02 & $1.01-1.04$ & 0.010 \\
\hline Intravenous induction & 1.12 & $1.01-1.56$ & 0.012 \\
\hline High BP & 6.47 & $5.97-7.03$ & $<0.001$ \\
\hline $\mathrm{BMI} \geq 85$ th percentile & 1.22 & $1.13-1.33$ & $<0.001$ \\
\hline OSA history & 1.51 & $1.32-1.72$ & $<0.001$ \\
\hline ASA 1 and 2 vs 3 & 1.39 & $1.28-1.52$ & $<0.001$ \\
\hline \multicolumn{4}{|l|}{ Pseudo $R^{2}=0.14$} \\
\hline \multicolumn{4}{|c|}{$\begin{array}{l}\text { Hosmer-Lemeshow test }=\text { chi-square }=10.51, \mathrm{df}=8 ; P=0.23 \\
n=35832\end{array}$} \\
\hline \multicolumn{4}{|l|}{ Final model } \\
\hline High BP & 6.22 & $5.76-6.71$ & $<0.001$ \\
\hline $\mathrm{BMI} \geq 85$ th percentile & 1.22 & $1.13-1.32$ & $<0.001$ \\
\hline OSA history & 1.49 & $1.31-1.32$ & $<0.001$ \\
\hline ASA 1 and 2 vs 3 & 1.39 & $1.28-1.52$ & $<0.001$ \\
\hline
\end{tabular}

OSA, obstructive sleep apnea; BMI, body mass index; BP, blood pressure; ASA, American society of anesthesiologists; OR, odds ratio; $\mathrm{Cl}$, confidence interval.

High BP in children is an increasingly prevalent public health problem that is frequently overlooked by clinicians $(6,24)$. Our finding that one-third of children in the present study had elevated BP measurements is concerning for clinical and epidemiological reasons. First, these BP measurements were measured in apparently healthy children prior to undergoing elective surgery and anesthesia thus reducing the confounding influence of pain on the $\mathrm{BP}$ readings. Thus, the high prevalence of HBP is likely a reflection of the secular trend in high $\mathrm{BP}$ among children especially those who are overweight/obese. Because there was no indication (based on documentation) that these blood pressures were reassessed even in children with elevated BP by adult standards, there is a need for increased awareness of the rising prevalence of HBP in children.

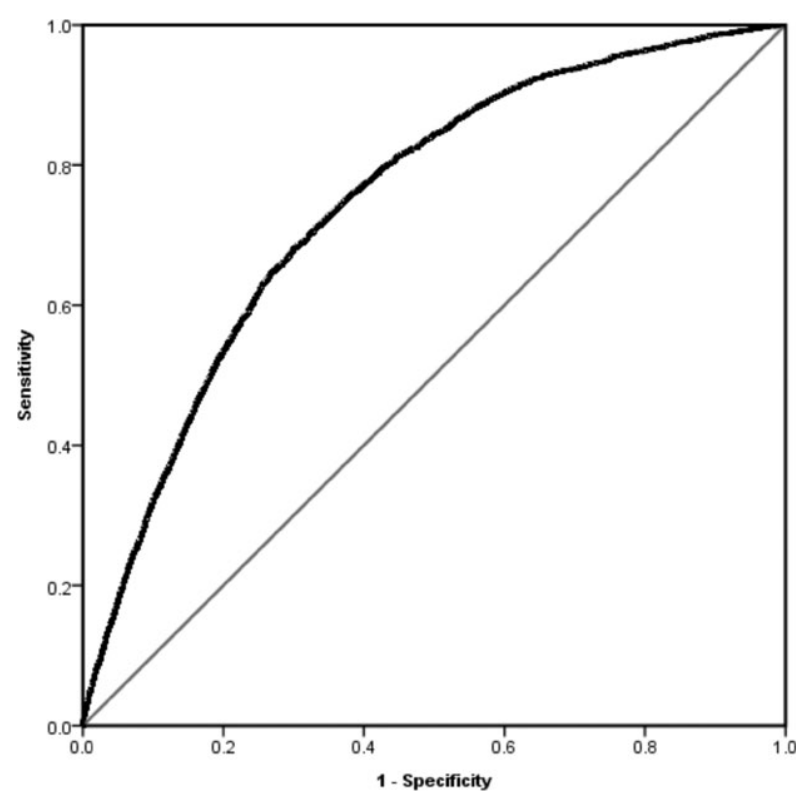

Figure 3 Receiver operator characteristic (ROC) curve evaluating the sensitivity and specificity of our model predicting multiple episodes of $\mathrm{PIH}$. Area under the curve $(\mathrm{AUC})$ for the predictors was $0.75 ; 95 \% \mathrm{Cl}=0.73-0.77 ; P=0.001$. AUC for the ROC indicates the usefulness of a test (our model) in predicting a binomial outcome (multiple hypotension yes/no). A value of 0.75 is a 'fair' predictive ability for our model.

Furthermore, because the preoperative environment represents a health care visit, children should have their BP measured and those with elevated readings should have this reassessed. Additionally, persistently elevated BP in the preoperative setting could be used as a teachable moment (24) for the dissemination of impactful health information. This could be targeted particularly at children in the high BMI category. It is highly conceivable that parents or caregivers of an obese hypertensive child will be receptive to health promotion information especially if the child has other obesityrelated comorbidities. Increasing childhood obesity rate 
is an important contributor to the growing prevalence of elevated BP $(9,10,13)$. Several epidemiologic and clinical reports $(9,25,26)$ describe the strong association of overweight and obesity with high BP and prevalence of hypertension in childhood. Recent report on the secular trend of high BP in children indicates a rising prevalence of elevated BP (prehypertension and hypertension) among children and adolescents of all ethnic groups in parallel with the increase in obesity prevalence, especially among minority children (12).

Accumulating data indicates that undiagnosed hypertension in children may not be entirely benign given its association with learning difficulties and cognitive dysfunction (27). It has been postulated that this cognitive abnormality may be due to abnormal cerebral vascular auto regulation. This observation suggests that a dismissive attitude toward high blood pressure in children is unwarranted and calls for active involvement of health care providers in the screening for and dissemination of preventive information about elevated BP to parents and children.

Although BP and other hemodynamic variables are routinely measured and closely monitored in the perioperative period, the clinical implications of elevated BP in the preoperative setting in children are unclear. Indeed elevated BP in the preoperative setting is likely to be overlooked and not reassessed because of a prevailing perceived negligible clinical impact on the perioperative outcome of children. Among adult surgical patients, undiagnosed or untreated high blood pressure (HBP) is often cited as a risk factor for perioperative complications (14-16). For example, adult hypertensive patients are prone to greater 'swings' in BP following induction and during laryngoscopy than their normotensive peers (15).

As another important finding of the present study, we report as hypothesized that elevated baseline BP is an independent predictor of multiple episodes of PIH in children undergoing elective noncardiac operations. To our knowledge, this is the first time high baseline BP is being shown to be an independent risk factor for intraoperative hypotension in children. Indeed, episodes of $\mathrm{PIH}$ were more frequent with increasing deciles of preoperative SBP. Although the longtime consequences of elevated BP are well known (26,28), this report provides the first intraoperative evidence of its association with postinduction hypotension in children. Furthermore, intraoperative hypotension may not necessarily be a benign complication in children given that it represents physiologic perturbation the effect of which is presently unknown. Clearly, any end-organ dysfunction following persistent intraoperative hypotension (28) is likely going to be attributed to the low BP thus emphasizing the importance of risk factors for intraoperative hypotension. Indeed, since the report of neurologic devastation in four infants with severe intraoperative hypotension (28), increasing attention is being given to intraoperative $\mathrm{BP}$ in children. We have not changed recommendations for intraoperative BP management at our institution in the age group reported in the present study. There is however heightened awareness of the importance of elevated preoperative BP among our care providers.

\section{Study limitations}

This study was conducted in one center, limiting the generalization of our finding to all children. Consistent with routine preanesthesia care, there was no way to independently assess the accuracy of the blood pressure measurements in the electronic medical record or the degree to which the blood pressure procedures were followed. However, single institution studies have the advantage of consistent, uniform, complete data collection as well as consistency of BP measurement. Additionally, BP in this study was measured with oscillometric methods rather than with auscultatory techniques, which are more rigorous and generally recommended (7). However, oscillometric monitors are used widely in routine clinical practice and are ideal for screening situation because they allow for multiple measurements in a noisy environment with less trained personnel. Our results are therefore consistent with what obtains in many preanesthesia screening facilities and may thus have wider applicability. Also, we had no record of preoperative anxiety status of the patients in the present study. Anxiety could potentially be responsible for the high rates of preoperative high $\mathrm{BP}$ in our patients. However, we are unaware of a biologically plausible reason why anxiety will predispose to higher rates of postinduction hypotension. Furthermore, we do not routinely use validated measures of preoperative anxiety to document anxiety status on our patients and we presume this to be the case in many busy pediatric anesthesia hospitals. Additionally, we did not collect data on preoperative use of anxiolytics. This could potentially increase the rates of postinduction hypotension. There is however no reason to suppose that children with baseline high BP would be more likely to receive preoperative anxiolytics. Indeed, given the association of obesity with high BP, it is highly plausible that obese patients would be less likely to have received preoperative anxiolytics thus reducing their possible confounding effects. Finally, the present study did not address the effect of weight-based dosing of medications such as opioids which could potentially affect the incidence of PIH. Our local practice is actual body weight- 
based dosing of most medications 'titrated to effect'. We cannot determine whether practitioners considered ideal or lean body weight in opioid dosing from the present study.

In summary, the present study indicates that baseline elevated BP was very common and strongly associated with childhood obesity status suggesting a group that may be targeted for preventive strategies. We also identified high preoperative BP as an independent risk factor for multiple episodes of PIH in children. Given the increasing recognition of intraoperative hypotension as a possible permissive factor for anesthesia-induced neurocognitive dysfunction, mechanisms underlying this hypotensive response deserve further elucidation.

\section{Funding}

This project was supported by the National Institute of General Medicine (NIGMS) at the National Institutes of Health (NIH) grant number K23 GM104354 (O.O. Nafiu). The views expressed in this publication are solely the responsibility of the authors and do not necessarily represent the official views of the NIH.

\section{Conflict of interest}

None of the authors have any conflict of interest to declare.

\section{References}

1 Din-Dzietham R, Liu Y, Bielo MV et al. High blood pressure trends in children and adolescents in national surveys, 1963-2002. Circulation 2007; 116: 1488-1496.

2 McNiece KL, Poffenbarger TS, Turner JL et al. Prevalence of hypertension and prehypertension among adolescents. $J$ Pediatr 2007; 150: 640-644.

3 Sorof JM, Lai D, Turner J et al. Overweight, ethnicity, and the prevalence of hypertension in school-aged children. Pediatrics 2004; 113 : 475-482.

4 Moore WE, Stephens A, Wilson T et al. Body mass index and blood pressure screening in a rural public school system: the Healthy Kids Project. Prev Chronic Dis 2006; 3: A114.

5 Dietz WH. Health consequences of obesity in youth: childhood predictors of adult disease. Pediatrics 1998; 101: 518-525.

6 Lande MB, Carson NL, Roy J et al. Effects of childhood primary hypertension on carotid intima media thickness: a matched controlled study. Hypertension 2006; 48: 40-44.

7 Elias MF, Elias PK, Sullivan LM et al. Lower cognitive function in the presence of obesity and hypertension: the Framingham heart study. Int J Obes Relat Metab Disord 2003; 27: 260-268.

8 Adams HR, Szilagyi PG, Gebhardt L et al. Learning and attention problems among children with pediatric primary hypertension. Pediatrics 2010; 126: e1425-e1429.

9 Hansen ML, Gunn PW, Kaelber DC. Under-diagnosis of hypertension in children and adolescents. JAMA 2007; 298: 874-879.

10 National High Blood Pressure Education Program Working Group on High Blood Pressure in Children and Adolescents. The fourth report on the diagnosis, evaluation, and treatment of high blood pressure in children and adolescents. Pediatrics 2004; $\mathbf{1 1 4}$ (2 suppl 4th report): 555-576.

11 Apfelbaum JL, Silverstein JH, Chung FF et al. Practice guidelines for postanesthetic care: an updated report by the American Society of Anesthesiologists Task Force on Postanesthetic Care. Anesthesiology 2013; 118: 291-307.

12 Rosner B, Cook N, Portman R et al. Blood pressure differences by ethnic group among United States children and adolescents. Hypertension 2009; 54: 502-508.

13 Sorof J, Daniels S. Obesity hypertension in children: a problem of epidemic proportions. Hypertension 2002; 40: 441-450.

14 Prys-Roberts C, Meloche R, Foëx P. Studies of anaesthesia in relation to hypertension. I. Cardiovascular responses of treated and untreated patients. Br J Anaesth 1971; 43: 122-137.

15 Prys-Roberts C, Greene LT, Meloche R et al. Studies of anaesthesia in relation to hypertension. II: hemodynamic consequences of induction and endotracheal intubation. $\mathrm{Br}$ $J$ Anaesth 1998; 80: 106-122.

16 Wax DB, Porter SB, Lin HM et al. Association of pre-anesthesia hypertension with adverse outcomes. J Cardiothorac Vasc Anesth 2010; 24: 927-930.

17 Nafiu OO, Maclean S, Blum J et al. High BMI in children as a risk factor for intraoperative hypotension. Eur J Anaesthesiol 2010; 27: 1065-1068.

18 Nafiu OO, Kheterpal S, Morris M et al. Incidence and risk factors for preincision hypotension in a noncardiac pediatric surgical population. Pediatr Anesth 2009; 19: 232 239.

19 Reich DL, Wood RK Jr, Mattar R et al. Arterial blood pressure and heart rate discrepancies between handwritten and computerized anesthesia records. Anesth Analg 2000; 91: 612-616.

20 Coates BM, Vavilala MS, Mack CD et al. Influence of definition and location of hypotension on outcome following severe pediatric traumatic brain injury. Crit Care Med 2005; 33: 2645-2650.

21 Young IT. Proof without prejudice: use of the Kolmogorov-Smirnov test for the analysis of histograms from flow systems and other sources. J Histochem Cytochem 1977; 25: 935-941.

22 van der Schouw YT, Verbeek ALM, Ruijs JHJ ROC curves for the initial assessment of new diagnostic tests. Fam Pract 1992; 9: 506-511.

23 Nafiu OO, Zepeda A, Curcio C et al. Association of neck circumference and obesity status with elevated blood pressure in children. J Hum Hypertens 2014; 28: 263-268.

24 Flynn JT. Pediatric hypertension: recent trends and accomplishments, future challenges. Am J Hypertens 2008; 21: 605-612.

25 Chiolero A, Cachat F, Burnier M et al. Prevalence of hypertension in schoolchildren based on repeated measurements and association with overweight. $J$ Hypertens 2007; 25 : 2209-2217.

26 Salvadori M, Sontrop JM, Garg AX et al. Elevated blood pressure in relation to overweight and obesity among children in a rural Canadian community. J Pediatr 2008; 122: 821-827.

27 Brosgol Y, Kupferman JC, Goodman S et al Transcranial Doppler reactivity (TCR) abnormalities in pediatric hypertension: a potential biomarker for brain abnormalities. Ann Neurol 2009; 66(Suppl 13): S144.

28 McCann ME, Schouten AN, Dobija N et al. Infantile postoperative encephalopathy: perioperative factors as a cause for concern. Pediatrics 2014; 133: e751-e757. 